$2^{\circ}$ La centrifugation (Jean Perrin), qui permettrait d'arriver à un résultat analogue par une sorte d'écrémage fractionné des micelles suivant leur grosseur ;

$3^{\circ}$ Enfin, l'ultramicroscopie, qui permettrait peut-être même de déterminer par la méthode de Jean Perrin, le poids moléculaire des différents constituants et, si ce poids était le même, d'en conclure éventuellement à l'unité de la lactéine.

C'est là, en tous cas, la voie à suivre dans une nouvelle étude à entreprendre des matières albuminoïdes du lait, et plus généralement de tous les colloïdes organiques naturels.

$1^{\text {er }}$ Avril 1915 (1).

\title{
LES BESOINS EN BON LAIT D'UNE GONSULTATION DE NOURRISSONS. MOYENS D'Y SATISFAIRE.
}

\author{
par les Docteurs LANGEVIN et GRANVAL,
}

Médecins de la " Goutte de Lait du Mans ».

La direction d'une Goutte de Lait, c'est-à-dire d'une consultation de nourrissons avec distribution de lait, nécessite la solution d'un certain nombre de questions pratiques concernant le lait, qui se présentent dans chaque région avec quelques variantes et sur lesquelles nous désirons attirer l'attention des collaborateurs de la revue $\mathrm{Le}$ Lait. Puisse chacun d'eux nous aider - dans la partie technique qui lui est propre - à résoudre les difficultés auxquelles nous nous heurtons

(1) Cette étude a été écrite pendant la guerre, qui en avait jusqu'ici empéché la publication et l'auteur, mobilisé à cette époque, n'a qu'un mot à y ajouter actuellement : M. Jacques Duclaux a donné, dans le livre, dont une phrase a été mise après coup en tête de ce travail, un résumé théorique et pratique très complet de nos connaissances actuelles sur les colloïdes; toute personne qui désirera poursuivre l'étude des albuminoïdes du lait par les méthodes modernes, s'inspirera avec fruit des données de ce livre. Le journal Le Lait sera particulièrement heureux d'offrir ses colonnes aux travaux de ce genre, persuadé qu'il est, d'u re part de la fécondité de l'étude colloïdale du lait pour la compréhension des caractères " mystérieux » de ce dernier, et pour la connaissance exacte des albuminoïdes, substratum de la vie, d'autre part, de l'importance pratique des résultats qu'on est en droit d'attendre de cette étude pour la connaissance précise de l'opération de la coagulation, si importante dans les industries du lait (M. B.). 
dans l'approvisionnement en ben lait d'une consultation de nourrissons.

Evidemment l'idéal serait, nous semble-t-il, qu'à chaque Groutte de Lait soit annexée une vacherie scientifiquement installée, donnant un lait hygiénique.

Ces installations ne peuvent être, sáuf exception, faites à l'heure actuelle que par les municipalités. Depuis la guerre, l'état de leurs finances ne le leur permet pas.Nous ne savons guère imaginer d'autre solution parfaite, l'indigence de notre législation actuelle concernant le lait ne nous permettant pas d'espérer, en toute certitude, d'un lait commercial, les qualités nécessaires pour l'alimentation du nourrisson.

Au Mans, à la "Goutte de Lait » que nous dirigeons depuis 1 " ans, nous avons dû toujours nous accommoder de moyens de fortune, devenus même plus précaires encore pendant la guerre et pendant la période présente d'après guerre, par suite de la rareté et de la cherté du lait. Nous allons seulement exposer ici ce que nous avons fait, avec quelles imperfections et diffieultés. Enfin, nous donnerons les résultats auxquels nous arrivons, tant au point de vue sanitaire qu'économique.

Nous avions à fournir le lait quotidien à une moyenne de $\mathbf{1 5 0}$ enfants, depuis leur naissance jusqu'à 14 mois. Faute d'autres moyens et pourtant soucieux de nous adresser directement au producteur, nous avons dû demander ce lait aux cultivateurs des environs immédiats de la ville. Autour du Mans, par suite du morcellement des cultures par 4 à $\breve{~ h e c t a r e s, ~ l e s ~ e x p l o i t a n t s ~ d i s p o s e n t ~ s e u l e m e n t ~}$ en moyenne de 4 ou 5 vaches, rarement davantage. Nous avions donc souvent du lait de provenances multiples. Actuellement et depuis un an, nous avons pu - grâce à une convention avec un producteur, simplifier cette question d'origine. Il s'engage à répondre, chaque jour, à notre demande en lait. Il ne peut d'ailleurs le faire qu'en le collectant en partie dans son voisinage. En hiver, il nous apporte, le matin à la première heure, un lait comprenant deux traites, celle de la veille au soir et celle du matin, et nous avons obtenu qu'à la saison chaude, à partir du $1^{\text {er }}$ mai, il nous apporte dès le soir, le produit de la seconde traite.

Dans la région, la race laitière est représentée presqu'exclusivement par la vache normande. Race laitière excellente, dit-on, dont la production est évaluée de 2.800 à 3.000 litres en moyenne entre deux vêlages, pouvant donner à certaines époques de sa lactation jusqu'à 16 à 18 litres par jour d'un lait riche en matière grasse à $35 \%$ et même davantage.

En été, de juin, quelquefois de mars, jusqu'à novembre, elle vit. 
exclusivement au pâturage (prairies naturelles ou artificielles); en hiver, à l'étable, on Jui donne du foin sec, du son, des betteraves, des citrouilles. La culture de ces deux derniers produits est faite dans la région uniquement en vue de l'alimentation de la vache laitière. Le tout est accompagné de buvées tièdes.

La traite est faite deux fois par jour (matin et soir). Il est certain que la tenue des étables laisse beaucoup à désirer, que le pansage de ces animaux n'existe pas; néanmoins avant la traite le pis est quelquefois essuyé et même sonmis à un lavage sommaire. Le lait est reçu dans des récipients de métal ébouillantés et ensuite tamisé sur un linge propre.

Ce lait nous est apporté, dans des bidons du modèle courant,immédiatement après la traite du matin. Jusqu’à cette année, la "Goutte de Lait " ne recevait que du lait de la traite du matin et ce lait pouvait être immédiatement stérilisé. A cause de la pénurie du lait, nous avons dû accepter du lait de la traite du soir qui ne pourra être stérilisé qu'une demi journée après et pour la conservation duquel nous avons dô étudier un moyen de conservation. Nous avons choisi la glace de préférence à l'eau oxygénée. Les laitières ont reçu pour instructions, dès que leur traite est faite, de plonger les pots de lait dans l'eau froide, mais elles ne comprennent pas toujours la raison de ce refroidissement demandé et elles préfèrent le porter tiède à leur clientèle pour lui prouver que le lait vient d'être trait. Nous avons dû lutter contre cette erreur de manipulation.

Dès l'arrivée du lait à la " Goutte de Lait », l'embouteillage commence immédiatement. Le lait est de nouveau tamisé sur un linge fin, placé entre deux tamis métalliques. Après les coupages et sucrages nécessaires, la séparation en flacons à têtée est faite avec soin. Les flacons, répartis dans leurs paniers métalliques, sont mis à l'autoclave, coiffés de leurs bouchons caoutchoutés, mais non bouchés. Ils sont portés à $10 \mathrm{\Xi}^{\circ}$ pendant 40 minutes. Nous avons été obligés de ne pas mettre les flacons bouchés dans les étuves, la casse dans ce cas étant extrêmement considérable. Les bouchons sont appliqués sur les bouteilles dès la sortie des paniers de l'étuve, alors que la température du lait est encore au moirs de $80^{\circ}$. Des recherches microbiologiques ont montré que ce lait était parfaitement stérilisé. Les paniers ainsi préparés, conservés jusqu'au lendemain matin, sont distribués, par petites voitures, dans les divers quartiers de la ville ou pris directement à la " Goutte de Lait».

Pour certains enfants inaptes à digérer le lait de vache, il est préparé un lait maternisé qui donne fréquemment de bons résultats notamment chez les vomisseurs ou les enfants digérant mal la caséine. 
Des analyses de ce lait nous sont faites périodiquement parle Service des Fraudes du département. Malheureusement la réelle rareté actuelle du lait paralyse un peu les sanctions de ce Service. Nous-mẻmes nous apprécions la richesse en beurre à l'aide d'un crémomètre. Nous utilisons aussi un procédé qui nous a été indiqué par le Dr Dufour, de Fécamp, pour déceler l'état de pureté et de fraicheur de notre lait. Voici quels en sont le principe et la technique : si l'on ajoute à du lait quelques gouttes de carmin d'indigo, pour donner au liquide un teint bleu pâle, on s'aperçoit au bout de quelque temps, que cette coloration se détruit. Duclaux, qui le premier a attiré l'attention sur ce phénomène, l'attribue aux bactéries de l'air. Ces bactéries que le lait contient, dès l'origine, sont aérobies et mani festent leur action réductrice en transformant la matière colorante bleue en indigo blanc.

Dans un flaçon de $100 \mathrm{~cm}^{3}$ à large ouverture, on verse avec un compte-gouttes normal, einq gouttes d'une solution d'indigotine pure au millième, ou de bleu de méthylène au millième, on remplit avec du lait à examiner; on bouche hermétiquement, au moyen d'un bouchon paraffiné et on conserve à la lumière diffuse.

Il faut tenir compte de la température ambiante et voici les règles de temps minimum pendant lequel cet échantillon de lait reste coloré :

12 heures au-dessous de $10^{\circ}$;

8 - de 15 à $20^{\circ}$;

4 - au-dessus de $20^{\circ}$.

Grâce à ee procédé, nous savons rapidement, surtout en été, où la chose est capitale, si le lait qu'on nous a livré est bien de la traite du matin, comme nous l'exigeons. $\mathrm{Si}$, par aventure, on avait fait des coupages du lait, l'eau de la ferme étant impure, nous le saurions de suite.

Nous ne nous dissimulons pas qu'une pareille faęon de proıséder ne répond pas dans l'ensemble et d'une façon absolue aux exigences en bon lait du nourrisson. D'ailleurs les écueils, les difficultés que nous rencontrons, nous le rappellent assez fréquemment.

Une première difficulté, encore présente, résulte de la rareté du lait. Dans notre région - comme sans doute dans le reste de la France - elle tient à beaucoup de causes nées de la guerre. C'est la diminution du cheptel national par suite des réquisitions militaires qui ont porté, en maintes occasions, même sur le bétail laitier sacrifié pour les besoins de nos armées. C'est l'élévation du prix de la viande, entrainant le sacrifice des veaux femelles malgré les interdictions et par suite d'un contròle impossible des tueries rurales, le prix très élevé des vaches laitières, les rendant inaccessibles à 
beaucoup de petits cultivateurs, - la taxation du lait par arrétés préfectoraux à des prix inférieurs au désir du producteur, - l'installation de beurreries et fromageries aux environs immédiats de la ville - la diminution du fourrage par suite de la réquisition, - la fièvre aphteuse qui a sévi autour du Mans depuis plus d'une année, enfin l'absence de moyens légaux de réquisition du lait pour l'usage des enfants et des malades.

Parallèlement et comme conséquence naturelle à cette disette générale du lait, nous avons été surpris par un accroissement rapide de notre elientèle de nourrissons, les parents ne pouvant pas se procurer du lait dans leur voisinage.

Quoi qu'il en soit et malgré ces difficultés, nous avons pu presque toujours réponure aux demandes par un approvisionnement en quantité suffisante.

Mais il faut avouer que ce ne fut pas toujours un lait de première qualité.

A notre retour des armées, à la fin de l'hiver 1918-1919, nous avons été très surpris en constatant que les rations dont nous usions avant la guerre, ne produisaient pas les accroissements de poids que nous avions l'habitude d'obtenir. Nous nous sommes alors rendu compte par des analyses que la teneur de ces laits en principes nutritifs était à la limite inférieure permise.

Comme conséquence, nous avons dû augmenter légèrement nos rations. Les causes de cet état de choses étaient peut-être dans des falsifications, mais aussi dans l'insuffisance des rations alimentaires du bétail. Peu de fourrage d'abord, du son provenant de blé épuisé à 85 ou $88 \%$, ainsi qu'il était prescrit pendant la guerre et donné à cause de son prix élevé en petite quantité, l'usage presque exclusif de paille d'avoine à certaines époques.

La qualité insuffisante de l'alimentation du bétail a paru expliquer encore certains troubles digestifs, tels que diarrhées constatées avec fréquence à des époques non estivales, notamment en automne. Nous sommes enclins à penser qu'il faut incriminer la nourriture des vaches laitières faite souvent dans notre région à ces époques de betteraves et citrouilles. II a déjà été signalé que des produits toxiques venus de ces aliments non décelables par l'analyse, résistent à une stérilisation très exacte.

Enfin, il est assez fréquent dans la saison chaude que nous trouvions des échantillons de lait qui « tourne ». L'installation primitive des étables dans la région, l'absence totale de pansage des animaux, la méconnaissance fréquente des précautions élémentaires de la traite et du transport du lait nous paraissent expliquer suffisamment la souillure du lait et son acidification. Nous n'avons pu encore pour y 
remédier dans la mesure du possible que réclamer l'apport immédiat en hiver de la traite du matin - avec celle de la veille au soir dans des récipients séparés - en été l'apport biquotidien du lait et la conservation de la traite du soir à la Goutte de Lait dans des glacières.

Il est arrivé deux fois en 19 ans d'avoir quelques biberons qui ont subi une fermentation putride gazeuse, sans coagulation du lait. Cette fermentation se produisait dans les journées très chaudes et nous n'avons pu en attribuer la cause qu'à des anaérobies peptonisants dont notre stérilisation habituelle à $105^{\circ}$ ne détruit pas les spores.Au point de vue sanitaire, quoique le lait que nous distribuons ne puisse être considéré comme un lait hygiénique pour nourrissons, l'influence de notre ouvre reste excellente, la moyenne de la mortalité par gastroentérite ayant été pendant ces vingt dernières années de 1 à $2 \%$, alors que la mortalité correspondante pour la ville du Mans, quoique déjà améliorèe par le contingent appartenant à la Goutte de Lait, était encore d'environ $7, \mathrm{~b} \%$.

La mortalité générale a été de 2 à $3 \%$ à la Goutte de Lait, alors qu'elle était de $17,5 \%$ dans le, reste de la ville.

Pendant l'année 1920, sur 360 enfants qui sont passés à la Goutte de Lait, il n'en est mort aucun de gastro-entérite.

A l'heure où nous devons tous nous préoccuper du relèvement de la natalité et de l'aceroissement de la population, le prix du lait prend une importance particulière. Les Gouttes de Lait ont besoin de ressources singulièrement élevées pour remplir leur rôle humanitaire. Le budget de la Goutte de Lait du Mans qui, avant la guerre, était d'environ 15.000 franes, a atteint cette année 79.000 franes. Le lait nous est vendu, suivant la saison, 70 et 80 cent. le litre. Nous fournissons chaque ration quotidienne à $0 \mathrm{fr}$. 50 pour les indigents et 0 fr. 75 pour les demi-payants.

Nous sommes loin des années d'avant guerre où le lait ne valait

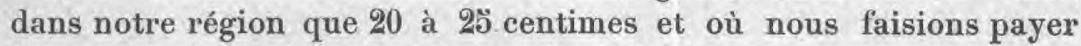
10 centimes la ration quotidienne des indigents.

Si nous considérons le coût d'un enfant indigent nous voyons que sa nourriture en lait représente pour sa famille, dans sa première année, environ 200 francs, chiffre que nous pouvons doubler pour l'enfant aisé.

D'ailleurs avec les frais généraux, les salaires élevés du personnel, le prix considérable de la verrerie, du eaoutchouc, du charbon, chaque ration de lait nous est revenue en 1920 à $1 \mathrm{fr} .60$.

Nous nous rendons pleinement compte par ces imperfections que nous sommes loin du " bon lait ", du lait hygiénique, le seul que nous voudrions fournir aux nourrissons. Les qualités que nous lui 
voudrions, d'être : $1^{\circ} \mathrm{pur}$, complet, de richesse modérée; $2^{\circ}$ sain, indemne de microorganismes, en particulier de tuberculose, de subs. tances toxiques de provenances diverses; $3^{\circ}$ bien conservé ; $4^{\circ}$ enfin d'un prix réduit, peuvent certainement ètre obtenues. Les résultats acquis dans d'autres pays sous un contrôle scientifique autorisent à le penser. Mais en France, à l'heure et dans les conditions où nous sommes, elles nous paraissent impossibles à réaliser.

Il est vraisemblable qu'un avenir prochain, dégagé du malaise économique actuel, améliorera la situation au moins au point de vue pécuniaire. Mais, à notre sens, aucune solution parfaite ne pourra être donnée à cette question du besoin en bon lait d'une consultation de nourrissons, avant que des vacheries modèles adaptées à ce but n'aient été créées et qu'une législation précise et rigoureuse du lait n'ait assuré en France le contrỏle de sa qualité.

\section{BIBLIOGRAPHIE ANALYTIQUE}

\section{$1^{\circ}$ LIVRES.}

A.-B. Marfan, Professeur de Clinique infantile à la Faculté de Médecine de Paris.- Traité de l'Allaitement, $3^{\circ}$ édit. in $-8^{\circ}$ de 920 p., 1921, 45 fr., Masson et Cie. éditeurs.

C'est avec le plus grand plaisir que nous faisons part à nos lecteurs de l'apparition de la $3 \bullet$ édition, depuis longtemps attendue, du livre bien connu du Professeur Marfan, collaborateur de cette revue, sur l'allaitement.

C'est une œuvre considérable, moins encore par son étendue, car le volume approche de 1.000 pages, que par le grand soin que l'auteur a apporté dans sa rédaction.

On y trouve derrière une convaissance parfaite du sujet, un grand esprit critique, nulle hésitation à aborder les difficultés, une application soutenue à chercher à les résoudre par la discussion de toutes les hypothèses qui se présentent, et l'apport bien choisi des arguments propres à soutenir tel ou tel point de vue.

C'est une ouvre très personnelle en même temps que fortement nourrie, qui fait honneur, non seulement à son auteur, mais à la littérature médicale française tout entière.

L'ouvrage comporte quatre parties :

10 Le lait, la digestion, les échanges nutritifs chez l'enfant du premier âge ; 